\title{
The combination of an inflammatory peripheral blood gene expression and imaging biomarkers enhance prediction of radiographic progression in knee osteoarthritis
}

Mukundan Attur ${ }^{1,2^{*+}}$ D , Svetlana Krasnokutsky ${ }^{1+}$, Hua Zhou ${ }^{3+}$, Jonathan Samuels ${ }^{1}$, Gregory Chang ${ }^{4}$, Jenny Bencardino ${ }^{4,5}$, Pamela Rosenthal ${ }^{1}$, Leon Rybak ${ }^{3}$, Janet L. Huebner ${ }^{6}$, Virginia B. Kraus ${ }^{6}$ and

Steven B. Abramson ${ }^{1}$

\begin{abstract}
Objective: Predictive biomarkers of progression in knee osteoarthritis are sought to enable clinical trials of structure-modifying drugs. A peripheral blood leukocyte (PBL) inflammatory gene signature, MRI-based bone marrow lesions (BML) and meniscus extrusion scores, meniscal lesions, and osteophytes on $\mathrm{X}$-ray each have been shown separately to predict radiographic joint space narrowing (JSN) in subjects with symptomatic knee osteoarthritis (SKOA). In these studies, we determined whether the combination of the PBL inflammatory gene expression and these imaging findings at baseline enhanced the prognostic value of either alone.

Methods: PBL inflammatory gene expression (increased mRNA for IL-1 $\beta$, TNFa, and COX-2), routine radiographs, and 3 T knee MRI were assessed in two independent populations with SKOA: an NYU cohort and the Osteoarthritis Initiative (OAI). At baseline and 24 months, subjects underwent standardized fixed-flexion knee radiographs and knee MRI. Medial JSN (mJSN) was determined as the change in medial JSW. Progressors were defined by an mJSN cut-point ( $\geq 0.5 \mathrm{~mm} / 24$ months). Models were evaluated by odds ratios (OR) and area under the receiver operating characteristic curve (AUC).

(Continued on next page)
\end{abstract}

\footnotetext{
* Correspondence: Mukundan.Attur@nyulangone.org

${ }^{\dagger}$ Mukundan Attur Svetlana Krasnokutsky Hua Zhou are co-first authors and contributed equally to work.

'Department of Medicine, NYU Grossman School of Medicine, New York, NY, USA

${ }^{2}$ Division of Rheumatology, Rheumatology Research laboratory, NYU Langone Orthopedic Hospital, 301 East 17th Street, Suite 1612, New York, NY 10003, USA

Full list of author information is available at the end of the article
}

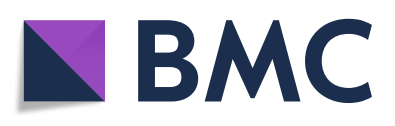

(- The Author(s). 2020 Open Access This article is licensed under a Creative Commons Attribution 4.0 International License, which permits use, sharing, adaptation, distribution and reproduction in any medium or format, as long as you give appropriate credit to the original author(s) and the source, provide a link to the Creative Commons licence, and indicate if changes were made. The images or other third party material in this article are included in the article's Creative Commons licence, unless indicated otherwise in a credit line to the material. If material is not included in the article's Creative Commons licence and your intended use is not permitted by statutory regulation or exceeds the permitted use, you will need to obtain permission directly from the copyright holder. To view a copy of this licence, visit http://creativecommons.org/licenses/by/4.0/. The Creative Commons Public Domain Dedication waiver (http://creativecommons.org/publicdomain/zero/1.0/) applies to the data made available in this article, unless otherwise stated in a credit line to the data. 
(Continued from previous page)

Results: We validated our prior finding in these two independent (NYU and OAI) cohorts, individually and combined, that an inflammatory PBL inflammatory gene expression predicted radiographic progression of SKOA after adjustment for age, sex, and BMI. Similarly, the presence of baseline BML and meniscal lesions by MRI or semiquantitative osteophyte score on $X$-ray each predicted radiographic medial JSN at 24 months. The combination of the PBL inflammatory gene expression and medial BML increased the AUC from $0.66(p=0.004)$ to $0.75(p<$ $0.0001)$ and the odds ratio from 6.31 to $19.10(p<0.0001)$ in the combined cohort of 473 subjects. The addition of osteophyte score to BML and PBL inflammatory gene expression further increased the predictive value of any single biomarker. A causal analysis demonstrated that the PBL inflammatory gene expression and BML independently influenced mJSN.

Conclusion: The use of the PBL inflammatory gene expression together with imaging biomarkers as combinatorial predictive biomarkers, markedly enhances the identification of radiographic progressors. The identification of the SKOA population at risk for progression will help in the future design of disease-modifying OA drug trials and personalized medicine strategies.

Keywords: Osteoarthritis, Radiographic progression, Joint space narrowing, Inflammatory gene expression, Molecular biomarkers, Bone marrow lesion, Magnetic resonance imaging

\section{Introduction}

Osteoarthritis (OA) is a leading cause of pain and morbidity globally, with increasing incidence and prevalence as the population ages [1]. OA is characterized by progressive and often relatively slow deleterious alteration of joint tissues, including cartilage, bone, and synovium [2-7]. Yet, no approved disease-modifying OA drugs (DMOADs) exist that slow progression of the disease. Radiographic progression of knee OA in unselected populations, measured as joint space narrowing (JSN), is low-approximately $0.1-0.15 \mathrm{~mm} /$ year [8-10]. However, in such studies, as many as $30-40 \%$ of the study population shows no evidence of JSN over 1-2 years, which presents a significant obstacle to DMOAD development [11-15]. To address this challenge, researchers have turned to identify baseline imaging and blood-based prognostic biomarkers that can differentiate progressors from non-progressors among patients with symptomatic knee OA (SKOA).

We have previously shown that increased peripheral blood leukocyte (PBL) gene expression of inflammatory proteins IL-1 $\beta, \mathrm{TNF} \alpha$, and COX-2 (PBL inflammatory gene expression) was associated with radiographic progression of knee OA at 24 months [15-19]. Similarly, separate studies indicate that the presence of bone marrow lesions (BML) on MRI is an imaging biomarker that identifies patients at higher risk for progression [20-22]. Although each of these biomarkers has demonstrated individual utility, no studies have focused on whether a combination of inflammatory and imaging biomarkers improves prediction of radiographic progression more than a single biomarker alone [23-27]. Maximizing the predictive value of baseline biomarkers will enable the powering and reduce the cost of future DMOAD studies [28].
The analyses reported here represent an extension of our existing cohort to 243 patients from a previous study of 111 patients, as well as an analysis of 204 SKOA patients selected from the OA Initiative (OAI). We determined the prognostic utility of the baseline PBL inflammatory gene expression, and MRI images, alone and in combination, as predictive biomarkers of SKOA radiographic progression. Additionally, we employed predictive multivariable models of a dichotomized outcome and used the area under the receiver operating characteristic curves (AUCs) to assess the predictive performance of baseline biomarkers to determine those most predictive of 24-month radiographic JSN. Our main aim was to identify baseline combinatorial biomarker(s) to predict and identification of patients at risk for "fast progression" of radiographic SKOA.

\section{Patients and methods \\ Patient population \\ NYU cohort}

Based on a priori knowledge, we expect a minimum of $30 \%$ of participants to progress (defined as JSN $\geq 0.5$ $\mathrm{mm} / 24$ months) and a minimum of $30 \%$ to show no evidence of progression (JSN $=0 \mathrm{~mm} / 24$ months. Therefore, we recruited 132 additional patients to our prior cohort of 111 patients with SKOA $(n=243)$ who completed a 24-month NIH-funded prospective study evaluating biomarkers in OA [11, 12] (Fig. 1), satisfying a power analysis to detect an effect size of 0.3 for biomarkers at significance level of 0.05 and power of 0.85 .

\section{NYU cohort inclusion and exclusion criteria}

As part of an NIH-funded study, we recruited and followed for 24 months SKOA patients who met American College of Rheumatology (ACR) knee OA 


\section{OAI SKOA Patients selection}

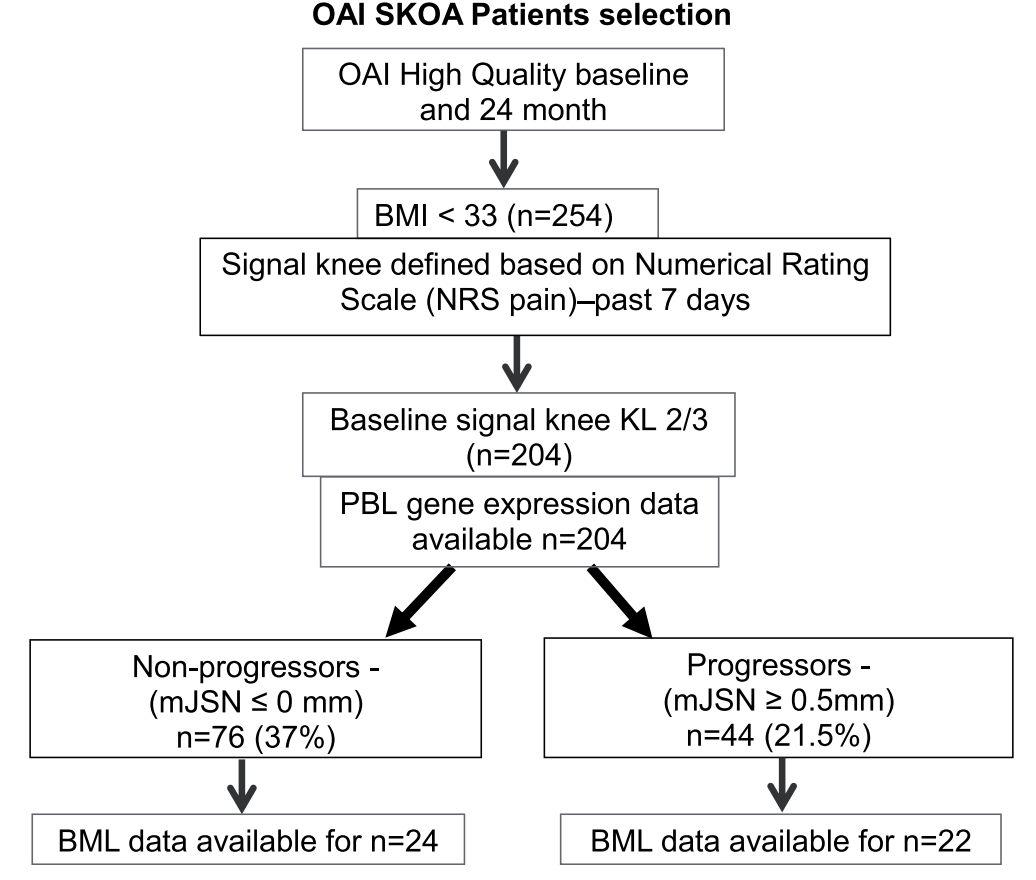

Fig. 1 Osteoarthritis Initiative symptomatic knee OA selection criteria for PBL gene expression and bone marrow lesion studies. PBL inflammatory gene expression data were available for all $(n=204)$ patients and MRI-BML data was available only for $n=46$ patients

radiographic criteria [Kellgren-Lawrence (KL) grade $\geq 1$ ] and clinical symptomatic criteria with at least 3 of the following: age $>50$ years, stiffness $<30 \mathrm{~min}$, crepitus, bony tenderness, bony enlargement, no palpable warmth [25]. Patients having any of the following were excluded: any other form of arthritis (including rheumatoid arthritis, spondyloarthritis, active crystal arthropathy); body mass index (BMI) $\geq 33$; any disorder requiring the use of systemic corticosteroids within 1 week of screening, history of bilateral knee replacements; major co-morbidities including diabetes mellitus, non-cutaneous cancer within 5 years of screening, chronic hepatic or renal disease, chronic infectious disease, congestive heart failure; and hyaluronan and/or corticosteroid injections to the affected knee within 3 months of screening. Some exclusion criteria, such as the BMI cutoff, were chosen to mitigate potential effects of the covariate on inflammatory peripheral blood leukocyte (PBL) gene expression markers, which were investigated as a separate aim of this study and reported upon elsewhere [17]. The Institutional Review Board at NYU Medical Center approved the protocol. Informed consent was obtained from all subjects.

\section{Clinical assessments}

All subjects had completed the visual analog scale (VAS) and WOMAC pain assessments at baseline and every 6 months for the duration of the study. Pain questions were specific to the more painful knee (signal knee).
Subjects also had a physical exam by a study physician at baseline and after 24 months.

\section{NYU imaging: radiograph and MRI acquisition and scoring \\ Knee radiographs}

Subjects underwent standardized weight-bearing fixedflexion posteroanterior knee radiographs using the SynaFlexer $^{\text {Tm }} \mathrm{X}$-ray positioning frame (Synarc) at baseline and 24 months as described previously [16, 29]. The radiographic beam angle was optimized for the medial joint space compartment. Radiographic readings were done separately by two musculoskeletal radiologists (LR, JB) blinded to patient demographics, clinical information, and MRI readings. X-rays were scored for KL grade $0-4$ [30], and medial and lateral joint space width (JSW) measured at the mid-portion of the joint space via electronic calipers. Joint space narrowing (JSN) was calculated as the change in JSW, in millimeters, from baseline to 24-month follow-up. Disagreements between the two readers were resolved by consensus. Cohen's kappa coefficient for interrater agreement on KL grades was 0.85 and 0.77 for the right knee and left knee, respectively, and kappas for JSW were $\geq 0.93$ for medial compartments of both the right and left knees. Based on the high inter-reader correlations, a single reader (LR) was employed for the 24-month follow-up. Osteophyte scoring-both medial and lateral osteophytes in tibial and femoral regions scored semiquantitatively $(0-3)[0=$ 
absent, $1=$ mild, $2=$ moderate, and $3=$ severe]. Since the majority of both the NYU and OAI subjects (approximately $80 \%$ ) had medial compartment disease, we restricted our analysis to medial radiographic JSN progressors.

\section{NYU knee MR imaging protocol}

Of the 243 subjects enrolled, only 111 subjects had MR imaging performed [on a 3.0 T clinical scanner (Magnetom Tim Trio; Siemens Medical Solutions, Erlangen, Germany) using an eight-channel transmit-receive phased-array knee coil (In vivo Corporation, FL)]. The knee imaging protocol consisted of a sagittal 3D-high resolution T1-weighted-fast low angle shot (FLASH) sequence with selective water excitation $(\mathrm{TR} / \mathrm{TE}=25 / 4 \mathrm{~ms}$; flip angle $=25 ; \mathrm{FOV}=15 \times 15 \mathrm{~cm}$; slice thickness $=1.5$ $\mathrm{mm}$; matrix $=512 \times 384$; receiver bandwidth $=200 \mathrm{~Hz}$ / pixel) as well as sagittal T2-weighted fat-saturated spin echo $(\mathrm{TR} / \mathrm{TE}=4000 / 75 \mathrm{~ms} ; \mathrm{FOV}=15 \times 15 \mathrm{~cm}$; slice thickness $=3 \mathrm{~mm}$; matrix $=256 \times 128$; receiver bandwidth $=130 \mathrm{~Hz} /$ pixel).

\section{NYU knee MR assessments: WORMS scoring}

A musculoskeletal radiologist (GC), blinded to the clinical and radiographic information, but not blinded to acquisition time point, performed Whole-Organ Magnetic Resonance Imaging Score (WORMS) scoring on sagittal T2-weighted fat-saturated images and sagittal T1weighted 3-D spoiled gradient-echo images [31]. Specifically, cartilage morphology (score of 0-6) and subarticular bone marrow lesions (BML; a score of 0-3) were scored within the anterior, central, and posterior regions of the medial and lateral tibial plateaus; central and posterior regions of the medial and lateral femoral condyles were also scored. Medial and lateral meniscal morphology (score of 0-4) were also evaluated. Interrater reliability was assessed by scoring 10 subjects in two separate sessions, 1 week apart. Paired $t$-tests, applied for assessing differences for cartilage, BML, and meniscus readings ( $p$ values $0.354,0.797$, and 0.766 for cartilage, BML and meniscus readings, respectively), showed that there were no significant differences, which verified reading reliability of our data. Cartilage medial scores were calculated as the sum of the medial femur central, medial femur posterior, medial tibia anterior, medial tibia central, and medial tibia posterior regions. Cartilage lateral scores were calculated as the sum of lateral femur central, lateral femur posterior, lateral tibia anterior, lateral tibia central, and lateral tibia posterior regions. Overall, cartilage scores were calculated by summing lateral and medial cartilage scores. BML medial scores were calculated as the sum of the medial femur central, medial femur posterior, medial tibia anterior, medial tibia central, and medial tibia posterior regions. BML lateral scores were calculated as the sum of lateral femur central, lateral femur posterior, lateral tibia anterior, lateral tibia central, and lateral tibia posterior regions. Overall, BML scores were calculated by summing lateral and medial BML scores.

\section{The OAl cohort}

The OAI is a multi-center, longitudinal, prospective observational study of knee OA. The main goal of the OAI is to develop a public domain research resource to facilitate the scientific evaluation of biomarkers for knee OA as potential surrogate endpoints for disease onset and progression. Participants were selected from the Osteoarthritis Initiative (OAI; http://www.oai.ucsf.edu/), a longitudinal cohort of 4796 participants with clinical, radiological, biochemical, and other data collected at baseline and annual follow-up visits. OAI recruited participants with SKOA, and also those with no OA but considered at high risk of incident OA. Clinical, radiographic, and MRI data were obtained from the OAI database (https://data-archive.nimh.nih.gov/oai). MRI images were scored for BMLs using the semiquantitative (SQ) (MRI Osteoarthritis Knee Score) MOAKS system available at the OAI site. For each subregion, MOAKS scores three features using an ordinal score for size, number of BMLs, and percentage of lesion that is a BML. The OAI dataset includes both MRI and radiographic images. Baseline clinical data, MRI BML scores, radiographs (baseline and 24 months), and buffy coat samples for PBL gene expression studies were obtained.

\section{OAI cohort: high-quality OAI (HQ-OAI) radiographs}

The OAI imaging acquisition techniques and reading protocols are publicly available at http://oai.epi-ucsf.org/ datarelease. Baseline OA severity was assessed on knee radiographs centrally read and graded according to the Kellgren-Lawrence (KL) system [30]. Briefly, bilateral posteroanterior fixed-flexion weight-bearing radiographic views were obtained using a SynaFlexerTM frame (Synarc, Newark, CA, USA). The detailed Radiographic Procedure Manual is available online (https://oai.epiucsf.org/datarelease/operationsManuals/

RadiographicManual.pdf). We selected a cohort of 443 cases, whose knee radiographs had high-quality MTP alignment (defined as the inter-margin distance (IMD) of $\leq 1.70 \mathrm{~mm}$ at baseline and 24-month films [32, 33]. Furthermore, from this high-quality sub-cohort, we have selected patients whose BMI is $<33$ and signal knee (painful knee) with KL 2 or $3(n=204)$ were selected for this study (Fig. 1).

MR image acquisition and quantitative measures Non-contrast MRIs were obtained on 3T Trio systems (Siemens Healthcare, Erlangen, Germany), and the 
complete pulse sequence protocol and sequence parameters have been described previously [34].

BML MOAKS Score MRI BML scores available for all the subjects for whom PBL inflammatory gene expression data available were downloaded from the OAI site. Briefly, BML was scored using the semiquantitative MRI Osteoarthritis Knee Score (MOAKS) system, which is available at the OAI site [35]. For each subregion, MOAKS scores three features using an ordinal score for size, number of BMLs, and percentage of lesion that is a BML. The utilization of study protocol and biospecimens were reviewed and approved by the NYU School of Medicine IRB.

Radiographic progression For the medial JSN outcome variable, our definition of radiographic progression was similar to the case definitions described previously based on previously published reports $[8,10,27]$. SKOA patients who had narrowing in the medial tibiofemoral compartment by at least $0.5 \mathrm{~mm}$ over 24 months from baseline in the signal knee. We defined non-progressors or no progression as no increase, defined as JSN $\leq 0.0$ mm over 24 months.

\section{Sample collection and assessment PBL isolation and inflammatory gene expression}

NYU cohort At the time of baseline knee radiographs, non-fasting blood samples were collected in pyrogenfree heparinized tubes for PBL isolation using the FicollHypaque density gradient centrifugation. Total RNA was isolated from PBLs and from citrate buffy coats (OAI cohort) using the Qiagen RNeasy Kit (Qiagen) as described previously $[15,17]$. For both NYU and OAI studies, relative expression of inflammatory mRNA expression in PBLs was determined using Predesigned TaqMan primer sets (IL1B - Hs00174097_m1; TNFA - Hs00174128 _m1; PTGS2 (COX-2) - Hs00153133_m1) (Applied Biosystems). qPCR was performed as previously described [15], normalized against housekeeping genes GAPDH and $18 \mathrm{~S}$, and fold-change was calculated using the delta $\mathrm{Ct}$ method [36]. For the OAI study, the relative foldchange data were calculated against super-control $(n=$ 100) obtained from OAI biorepository who did not develop knee OA over 8 years of follow-up and the qPCR obtained CT values of each target(s) including the housekeeping genes were shared with the OAI biorepository team for de-identification and the following association studies.

\section{Statistical methods}

The relationships between baseline clinical, demographic, and imaging variables, including Western
Ontario and McMaster Universities Osteoarthritis Index (WOMAC) pain, visual analog scale (VAS) pain, radiographic JSW, osteophytes, MRI-determined cartilage and BML scores, age, sex, and BMI were determined by Spearman's correlation. Associations between variables were assessed by partial correlations controlling for age, sex, and BMI. Support vector machines were used for building predictive multivariate models of a dichotomized outcome variable based on multiple biomarkers [37]. The null model against which all other predictive models are compared and $p$ values are calculated is the random model (i.e., coin-flipping model), and the random model has AUC of 0.5. For estimating future predictivity of multivariate predictive models, we used 10 -fold stratified cross-validation repeated with 100 different splits of data into 10-folds [38]. We used area under receiver operating characteristic curve (AUC) and logistic regression (either unadjusted or adjusted for age, sex, BMI) model to investigate the prognostic value of PBL gene expression, radiographic osteophytes, MRI$\mathrm{BML}$, and cartilage score for predicting JSN.

This multivariate method can fit both simple and complex functions, avoids overfitting by using effective regularization strategies, and has shown excellent empirical performance in a wide range of biomedical predictive modeling applications [39]. AUC values were compared against random models for significance using Delong's test [40]. False discovery rate (FDR) was used to adjust the $p$ values for multivariate comparison [41]. To evaluate whether medial BML as an additional predictor improved the regression model, we used 2 methods: (1) Delong's test comparing the AUCs from the model with biomarkers plus medial BML against the model with biomarkers alone, and (2) ANOVA comparing linear regression models of JSN with biomarkers plus medial BML versus biomarkers alone.

Causal graph analysis was performed, for which we used the FCI algorithm in the TETRAD software package (http://www.phil.cmu.edu/projects/tetrad/version 4.3.10-7). This method is capable of discovering a causal graph that most closely resembles the data distribution [9]. Independence testing was based on Fisher's $Z$ test, with the significance level set to 0.10 . No data manipulation of any kind (e.g., transformation, imputation, thresholding) was applied; therefore, these analyses were not biased toward particular causal hypotheses.

\section{Results}

An inflammatory PBL gene expression predicts fast radiographic progressors in both the NYU and OAI cohort We recruited a total of 243 SKOA patients, followed in the clinics of NYU and analyzed the PBL inflammatory gene expression as a predictive biomarker of radiographic progression. The baseline demographic and 
clinical characteristics of these subjects are summarized in Table 1. Of the 243 patients in the NYU cohort, 30\% exhibited $\geq 0.5 \mathrm{~mm} \mathrm{mJSN}$ at 24 months in the signal knee and were designated "fast progressors" [8, 10, 42]. PBL mRNA expression of IL-1 $\beta$, COX-2, and TNF $\alpha$, at baseline significantly predicted fast radiographic progressors with AUCs that ranged from 0.62 to $0.68(p=0.003$ to $<0.0001$ ) (Table 2).

To replicate these NYU Cohort PBL transcriptomic studies in an independent population, we next examined radiographic progression in an OAI Cohort $(n=204)$ of subjects with symptomatic knee osteoarthritis. "Fast progression" was observed in $22 \%$ of the OAI Cohort. In the NYU cohort, age as covariant did not have significant predictive power, whereas sex and BMI did have significant but moderate AUC values 0.62 and $0.61(p=0.001$ and 0.004 ) (Table 2) in predicting fast progressors. In contrast, in the OAI cohort, these variants did not achieve significance. We note that while age and BMI were similar in both cohorts, the OAI population had a higher percentage of females (NYU 33\%; OA 47\%). As shown in the combined cohort, both sex and BMI retained significance but with moderate predictive power in predicting fast progressors. Increased mRNA expression of IL-1 $\beta$, COX-2, and TNF $\alpha$, (levels between non-progressors and progressors are presented in supplemental Table 1) significantly predicted JSN fast progressors ( $\geq 0.5 \mathrm{~mm}$ at 2 years) with AUCs that ranged from 0.64 to $0.76(p=0.006$ to $<0.0001)$ (Table 2). In the combined NYU and OAI cohort of 447 subjects, the significance of an inflammatory PBL gene expression was retained (Table 2) in predicting fast radiographic progressors (AUC 0.66 to $0.67(p<0.0001)$ ).

\section{MRI cross-sectional imaging, radiographic, and symptom relationships}

The association of baseline MRI-scored variables with clinical and radiographic features at baseline and 2 years is shown in Table 3. Baseline medial tibial central BML scores associated significantly, but modestly, with baseline WOMAC scores for pain $(r=0.19, p=0.048)$ and 24-month VAS pain reports $(r=0.20, \quad p=0.043)$ (Table 3). Additionally, medial tibial central BML associated moderately with baseline $\mathrm{KL}$ scores and associated inversely with baseline JSW (Table $3, r=0.21, p<0.01$; $r=-0.22, p=0.018$, respectively). Cartilage scores in all WORMS medial subregions, separately and/or summed, were also associated with baseline KL scores $(r$ values ranging from 0.27 to 0.34 depending on the specific subregion, all $p$ values $<0.01$; Table 3 ). Similarly, cartilage scores in nearly all medial subregions were inversely associated with baseline JSW ( $r$ values ranging from -0.27 to -0.33 , all $p$ values $<0.01$ ). Meniscus overall readings were also associated with baseline WOMAC pain and

Table $1 \mathrm{NYU}$ and OAI cohorts SKOA subject baseline demographics and imaging features

\begin{tabular}{|c|c|c|}
\hline Variable & NYU $(\boldsymbol{n}=243)$ & OAI $(\boldsymbol{n}=203)$ \\
\hline Age (years) & $60.1(10.6)$ & $62.8(10.6)$ \\
\hline \multicolumn{3}{|l|}{ Sex (\%): } \\
\hline Male & $33.30 \%$ & $47.0 \%$ \\
\hline Female & $66.70 \%$ & $53.0 \%$ \\
\hline BMI $\left(\mathrm{kg} / \mathrm{m}^{2}\right)$ & $26.7(3.6)$ & $27.3(3.1)$ \\
\hline VAS $(0-100)$ & $42.3(29.9)$ & NA \\
\hline WOMAC (0-100) & $36.6(24.7)$ & $18.5(16.2)$ \\
\hline \multicolumn{3}{|l|}{ Radiographic joint space width (JSW) (mm): } \\
\hline Baseline & $3.65(1.34)$ & $3.62(1.30)$ \\
\hline 24 months & $3.13(1.51)$ & $3.36(1.49)$ \\
\hline \multicolumn{3}{|l|}{ Radiographic osteophytes: } \\
\hline Medial tibial plateau (MTP) (0-3) & $0.50(0.70)$ & $1.08(1.17)$ \\
\hline Medial femoral condyle (MFC) (0-3) & $0.87(0.69)$ & $0.93(1.02)$ \\
\hline Lateral tibial plateau (LTP) (0-3) & $0.41(0.64)$ & $0.99(0.80)$ \\
\hline Medial femoral condyle (LFC) (0-3) & $0.51(0.60)$ & $0.33(0.38)$ \\
\hline MRI: & $(n=111)$ & $(n=46)$ \\
\hline Mean medial BML WORMS score (0-15) & $1.13(1.86)$ & $1.90(2.04)$ \\
\hline
\end{tabular}

Data shown are the mean (SD), total number or percent affected, as indicated. SKOA symptomatic knee OA, BMI body mass index, VAS visual analog scale, WOMAC Western Ontario and McMaster Universities Osteoarthritis Index, WORMS Whole-Organ Magnetic Resonance Imaging Score, BML bone marrow lesion, NA not available. Both medial and lateral osteophytes in tibial plateau (MTP, LTP) and femoral condyle (MFC, LFC) regions scored semiquantitatively $(0-3)[0=a b s e n t$, 1-mild, 2-moderate, and $3=$ severe]. Subarticular bone marrow lesions (BML, a score of 0-3) were scored within the anterior, central, and posterior regions of the medial and lateral tibial plateaus; central and posterior regions of the medial and lateral femoral condyles were also scored 
Table 2 The area under the receiver operating characteristic curves (AUC) of inflammatory PBL gene expression biomarkers to radiographic fast progressors of JSN (>0.5 mm) at 24 months in patients with SKOA in NYU extended and OAI cohorts

\begin{tabular}{|c|c|c|}
\hline $\begin{array}{l}\text { Fast progressors (JSN }>0.5 \mathrm{~mm} \text { ) vs. } \\
\text { non-progressors (JSN } \leq 0.0 \mathrm{~mm} \text { ) }\end{array}$ & AUC (95\% Cl) & Adjusted $\boldsymbol{p}$ value for model \\
\hline \multicolumn{3}{|l|}{ NYU $(n=243)$} \\
\hline Age & $0.48(0.39-0.56)$ & 0.711 \\
\hline Sex & $0.62(0.54-0.71)$ & 0.001 \\
\hline BMI & $0.61(0.53-0.69)$ & 0.004 \\
\hline Age + sex + BMl (ASB) & $0.65(0.57-0.73)$ & $<0.0001$ \\
\hline$I L-1 \beta$ & $0.62(0.53-0.70)$ & 0.003 \\
\hline $\operatorname{cox}-2$ & $0.68(0.60-0.75)$ & $<0.0001$ \\
\hline TNFa & $0.66(0.57-0.74)$ & $<0.0001$ \\
\hline $\mathrm{IL}-1 \beta+\mathrm{COX}-2+\mathrm{TNFa}$ & $0.60(0.52-0.69)$ & 0.008 \\
\hline$I L-1 \beta+A S B$ & $0.65(0.57-0.73)$ & $<0.0001$ \\
\hline$C O X-2+A S B$ & $0.66(0.58-0.74)$ & $<0.0001$ \\
\hline TNFa + ASB & $0.64(0.56-0.72)$ & $<0.0001$ \\
\hline $\mathrm{IL}-1 \beta+\mathrm{COX}-2+\mathrm{TNF} a+\mathrm{ASB}$ & $0.66(0.58-0.74)$ & $<0.0001$ \\
\hline \multicolumn{3}{|l|}{ OAI $(n=203)$} \\
\hline Age & $0.38(0.27-0.49)$ & 0.987 \\
\hline Sex & $0.50(0.39-0.61)$ & 0.482 \\
\hline BMI & $0.38(0.27-0.48)$ & 0.989 \\
\hline Age + sex + BMl (ASB) & $0.36(0.26-0.47)$ & 0.995 \\
\hline $\mathrm{IL}-1 \beta$ & $0.76(0.66-0.85)$ & $<0.0001$ \\
\hline COX-2 & $0.64(0.53-0.75)$ & 0.006 \\
\hline TNFa & $0.68(0.58-0.79)$ & $<0.0001$ \\
\hline $\mathrm{IL}-1 \beta+\mathrm{COX}-2+\mathrm{TNFa}$ & $0.69(0.59-0.79)$ & $<0.0001$ \\
\hline$I L-1 \beta+A S B$ & $0.63(0.53-0.73)$ & 0.007 \\
\hline$C O X-2+A S B$ & $0.50(0.38-0.61)$ & 0.513 \\
\hline TNFa + ASB & $0.56(0.45-0.67)$ & 0.158 \\
\hline $\mathrm{IL}-1 \beta+\mathrm{COX}-2+\mathrm{TNFa}+\mathrm{ASB}$ & $0.59(0.48-0.69)$ & 0.063 \\
\hline \multicolumn{3}{|l|}{ NYU + OAI combined $(n=436)$} \\
\hline Age & $0.47(0.40-0.54)$ & 0.822 \\
\hline Sex & $0.56(0.49-0.63)$ & 0.039 \\
\hline BMl & $0.57(0.50-0.64)$ & 0.021 \\
\hline Age + sex + BMl (ASB) & $0.57(0.50-0.63)$ & 0.019 \\
\hline $\mathrm{IL}-1 \beta$ & $0.66(0.60-0.72)$ & $<0.0001$ \\
\hline COX-2 & $0.66(0.59-0.72)$ & $<0.0001$ \\
\hline TNFa & $0.67(0.60-0.73)$ & $<0.0001$ \\
\hline $\mathrm{IL}-1 \beta+\mathrm{COX}-2+\mathrm{TNFa}$ & $0.63(0.57-0.70)$ & $<0.0001$ \\
\hline$I L-1 \beta+A S B$ & $0.60(0.54-0.67)$ & 0.001 \\
\hline $\mathrm{COX}-2+\mathrm{ASB}$ & $0.59(0.53-0.66)$ & 0.003 \\
\hline TNFa + ASB & $0.57(0.50-0.64)$ & 0.019 \\
\hline $\mathrm{IL}-1 \beta+\mathrm{COX}-2+\mathrm{TNF} a+\mathrm{ASB}$ & $0.61(0.54-0.68)$ & 0.001 \\
\hline
\end{tabular}

$A S B$ age, sex, and BMI, JSN joint space narrowing, SKOA symptomatic knee OA, $A U C$ area under the (receiver operating characteristic) curve, $95 \% \mathrm{Cl} 95 \%$ confidence interval; All comparisons are versus non-progressors (JSN $\leq 0 \mathrm{~mm}$ ). NYU cohort: non-progressors $(n=115)$ and fast progressors $(n=72)$; OAl cohort: non-progressors $(n=76)$ and fast progressors $(n=44)$ 


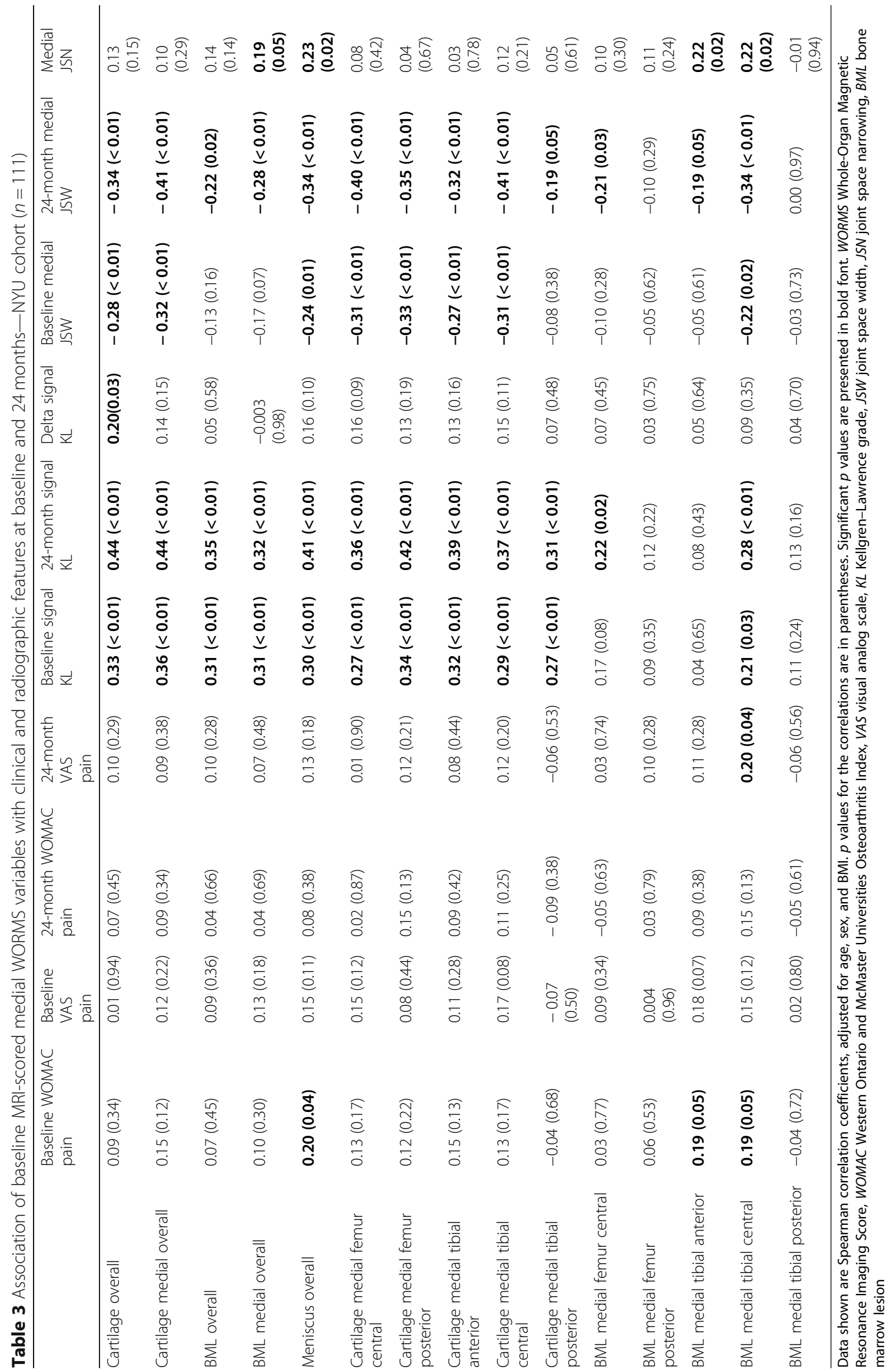


associated inversely with mJSW at 24 months $(r=-0.34$, $p<0.01)$.

\section{Baseline MRI medial BML (mBML) and meniscus scores predict the progression of radiographic findings}

BML detected by MRI has been associated with radiographic progression of knee OA [20-22, 43]. We analyzed MRI findings in our two OA cohorts to determine whether (1) MRI findings predicted the subset of fast progressors (JSN $>0.5 \mathrm{~mm}$ ). We note that in the NYU cohort, MRI was available for the initial 111 enrollees previously described [12]. Consistent with prior literature, fast progressors $(\geq 0.5 \mathrm{~mm})$ in the NYU cohort had significantly higher baseline medial BML scores $(1.78 \pm$ 2.22) than non-progressors $(0.59 \pm 1.14 ; \quad p<0.014$; Table 4A). Similarly, in the OAI cohort, medial BML scores were also higher in the fast progressors cohort (1.21 vs. $3.27 ; p<0.013$ ).

In the OAI cohort, "Cartilage Loss Medial" was also associated with progression, though this was not observed in the NYU cohort (Table 4B). In the combined cohort, association of medial BML alone with mJSN is also shown in Table 5 (AUC $=0.59$; 95\% CI $(0.51-0.67$; $p<0.035)$. The odds ratio (OR) for fast progression associated with medial BML was 2.43 (95\% CI 1.44-4.08; $p<0.0001$ ) (Table 6). In the NYU cohort, the MRI meniscus score correlated $(r=0.23 ; p=0.02)$ with mISN (Table 3), though AUC $(0.56 ; p=0.178)$ was modest and not significant in the dichotomized radiographic progression analysis (progressors vs. non-progressors). When meniscal findings were combined with BML and PBL inflammatory gene expression, the AUC for either alone increased significantly to $0.73 \quad(p<0.0001$; Table 7).
Combinatorial biomarkers (medial BML scores and PBL gene expression) enhance prediction of radiographic progression in the combined NYU and OAI cohorts Having shown that an inflammatory PBL transcriptome and BML by MRI individually predicted radiographic progression in our cohorts, we next determined whether any combination of biomarkers had greater predictive value than a single biomarker alone in the combined cohort.

We first examined the predictive value of the combination of baseline medial BML scores and PBL inflammatory gene expression markers by AUC analyses. As shown in Table 5, the association of age, sex, and BMI alone with fast progressors (JSN $\geq 0.5 \mathrm{~mm}$ ) was not significant with AUC ranging from 0.46 to 0.54 . Moreover, causal analysis (Fig. 2) did not show a direct effect of these variables on joint space narrowing. The association of BML alone with fast progressors (JSN $\geq 0.5 \mathrm{~mm}$ ) in the combined cohort was AUC $=0.59$ (95\% CI 0.51$0.67, p=0.035$; Table 5). In each instance, the combination of medial BML and individual PBL inflammatory gene expression increased the predictive value of either biomarker alone. Specifically, the combination of medial BML and PBL COX-2 expression (AUC 0.65, $p<0.0001$ ) yielded the maximal predictive power for fast progressors (Table 5). Additionally, the increased AUC model performance was significant for PBL mRNA transcripts (COX-2, IL1 $\beta$, and TNF $\alpha$ ) in combination with BML (Table 5) in predicting radiographic progression.

In addition to calculating the AUCs, we also determined the odds ratio (OR) for progression for each biomarker, alone and in combination. As shown in Table 6, the OR for baseline PBL COX-2 and IL- $1 \beta$ predicting fast progressors (JSN $\geq 0.5 \mathrm{~mm}$ ) were 3.56 and 1.90 respectively, and a combination of all three inflammatory

Table 4 Baseline cartilage and BML scores in radiographic non-progressors compared to fast progressors in NYU (4A) and OAI (4B) SKOA

\begin{tabular}{|c|c|c|c|c|}
\hline$A-N Y U$ & $\begin{array}{l}\text { Non-progressors JSN } \leq 0 \mathrm{~mm}(n=39) \\
\text { Mean (SD) }\end{array}$ & $\begin{array}{l}\text { Fast progressors JSN } \geq 0.5 \mathrm{~mm}(n=45) \\
\text { Mean (SD) }\end{array}$ & $p$ value & FDR \\
\hline Cartilage medial overall (0-30) & $13.88(8.61)$ & $15.42(8.71)$ & 0.42 & 0.42 \\
\hline Cartilage lateral overall (0-30) & $3.56(6.62)$ & $5.24(7.89)$ & 0.298 & 0.398 \\
\hline BML medial overall (0-15) & $0.59(1.14)$ & $1.78(2.22)$ & 0.003 & 0.014 \\
\hline BML lateral overall (0-15) & $0.33(0.80)$ & $0.16(0.47)$ & 0.214 & 0.398 \\
\hline B-OAI & $\begin{array}{l}\text { Non-progressors JSN } \leq 0 \mathrm{~mm}(n=29) \\
\text { Mean (SD) }\end{array}$ & $\begin{array}{l}\text { Fast progressors JSN } \geq 0.5 \mathrm{~mm}(n=34) \\
\text { Mean (SD) }\end{array}$ & $p$ value & FDR \\
\hline Cartilage loss medial (0-36) & $4.21(2.90)$ & $7.09(3.78)$ & 0.006 & 0.014 \\
\hline Cartilage loss lateral (0-36) & $5.04(4.09)$ & $2.32(2.80)$ & 0.012 & 0.025 \\
\hline BML medial overall (0-36) & $1.21(1.82)$ & $3.27(2.31)$ & 0.002 & 0.013 \\
\hline BML lateral overall (0-36) & $2.23(2.84)$ & $0.98(1.50)$ & 0.072 & 0.102 \\
\hline
\end{tabular}

Abbreviations: WORMS Whole-Organ Magnetic Resonance Imaging Score, BML bone marrow lesion, JSN joint space narrowing, FDR false discovery rate. JSN values are expressed in $\mathrm{mm}$ as mean \pm standard deviation (SD). All comparisons are versus non-progressors (JSN $\leq 0 \mathrm{~mm}$ ). Significant $p$ values are represented in bold font 


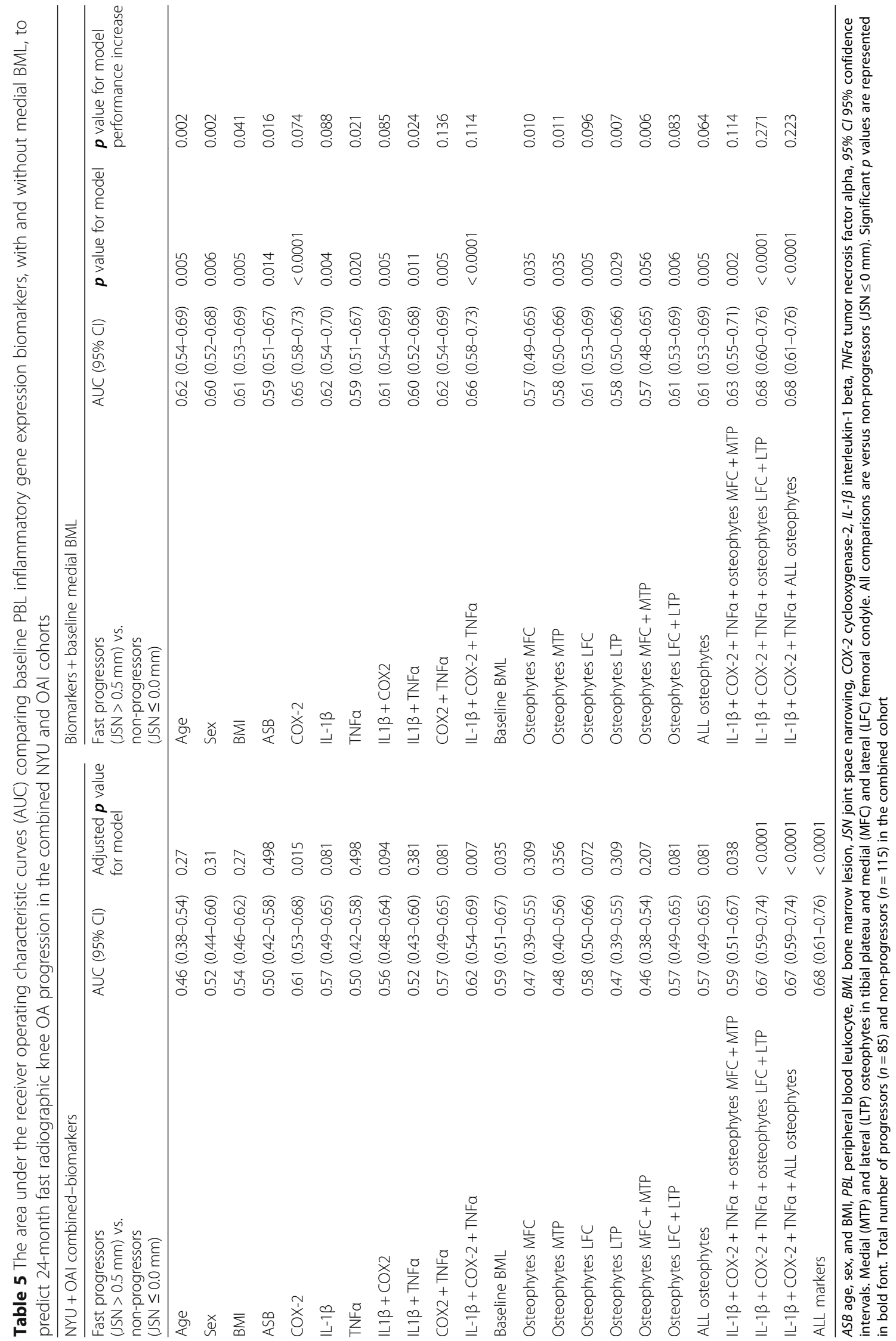




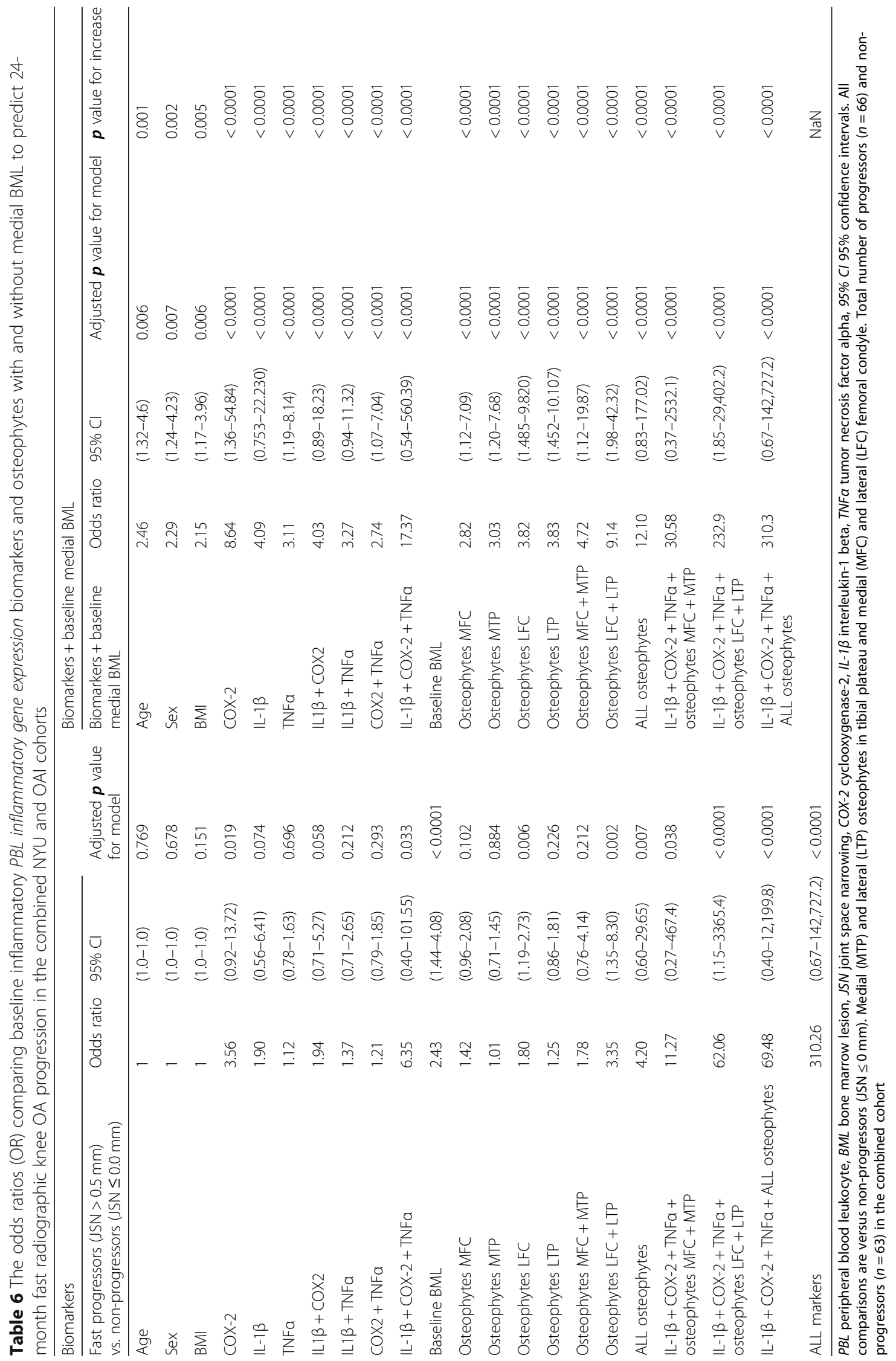




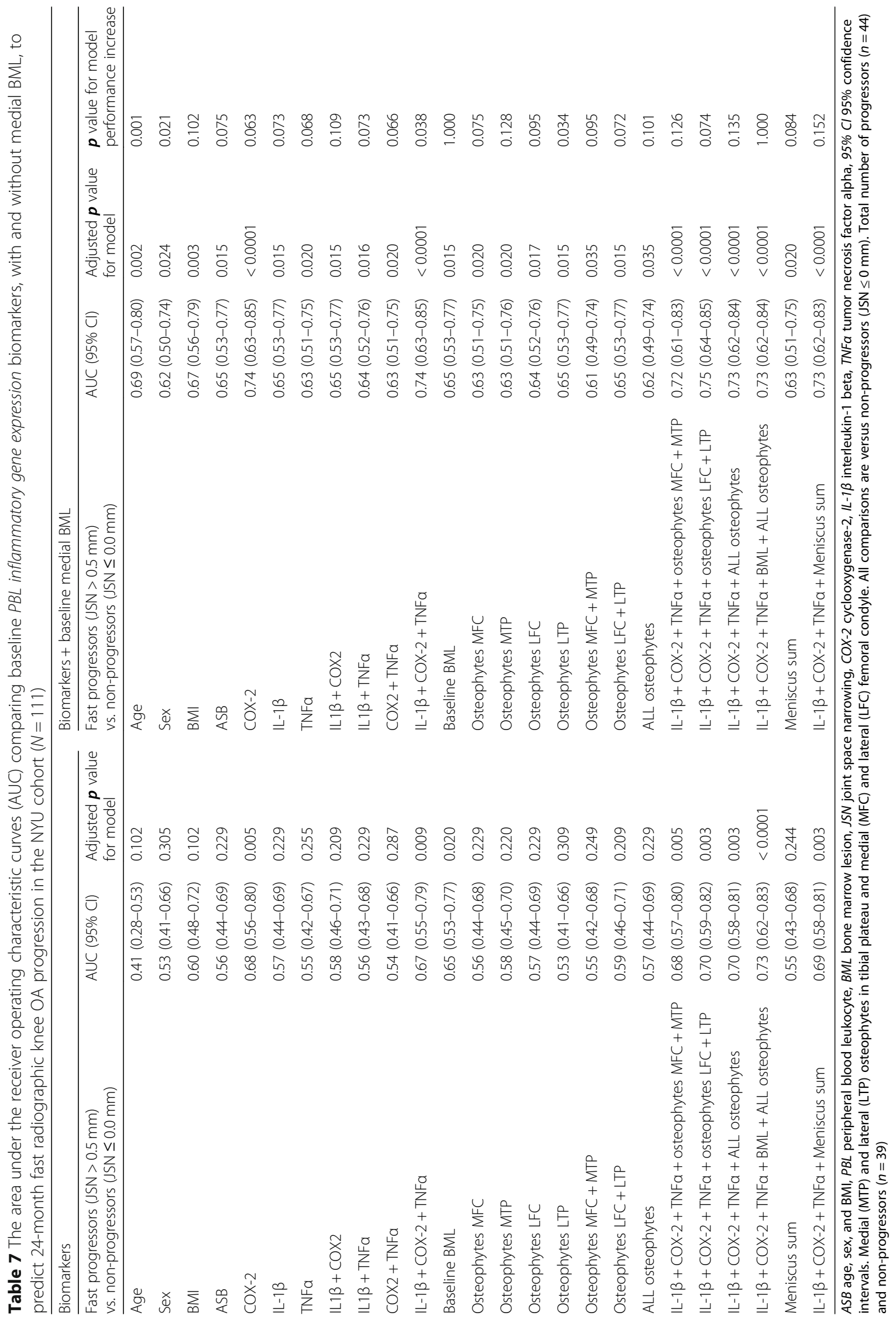




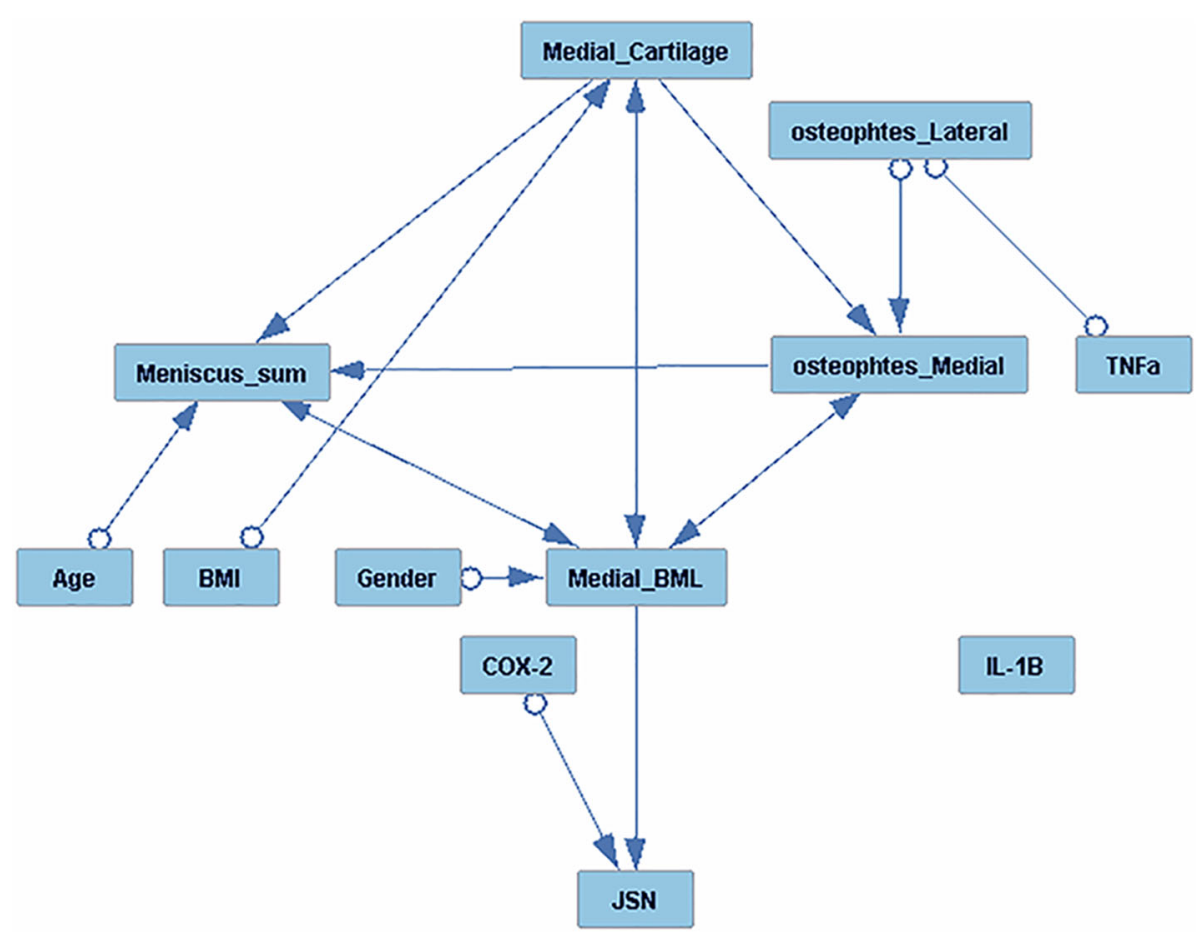

Fig. 2 Causal graph analysis of NYU cohort baseline, PBL inflammatory gene expression (molecular), X-ray (radiographic), and MRI biomarkers along with age, sex, and BMI on medial JSN. FCl algorithm was used for causal graph analysis of all variables, to determine the interdependence of, inflammatory PBL gene expression, radiographic (medial and lateral osteophytes) MRI imaging markers (medial BML, meniscus, and cartilage scores) and covariates (BMI, age, sex) on continuous radiographic JSN over 24 months. Edges with a single arrow denote causality, edges with double arrows denote hidden confounders, and marks (circles) on the edges denote uncertainty of causal orientation. JSN = joint space narrowing; PBL gene expression markers: IL-1 $\beta=$ interleukin-1 $\beta$; TNFa $=$ tumor necrosis factor $a$, and COX-2 = inducible cyclooxygenase-2

PBL gene expression biomarkers (IL-1 $\beta+$ TNF $\alpha+$ COX-2) had an OR of 6.35. The addition of baseline medial BML to inflammatory PBL IL-1 $\beta$, COX-2, and TNF $\alpha$ markers increased the OR significantly to 17.37 $(p<0.0001)$.

\section{Baseline radiographic osteophyte scores and progression of joint space narrowing}

Since osteophytes have also been reported to associate with radiographic progression in knee OA [44-47], we added osteophyte assessment to our predictive models in the combined cohort. As shown in Table 6, combination of medial and lateral osteophyte scores increased the OR for progression to $4.2(p=0.007)$. The combination of the PBL inflammatory gene expression and osteophyte markers increased the OR to 69.48 ( $p<$ 0.0001 ), while the combination of baseline BML, osteophytes, and molecular biomarkers further increased OR to $310.30(p<0.0001)$. Similarly, AUC analysis indicated that the predictive value of osteophytes alone significantly increased when combined with PBL gene expression or BML (Table 5).

\section{Causal graph analysis}

To further explore interactions of baseline radiographic (osteophytes) MRI features (medial BML, cartilage, and meniscus), and PBL inflammatory gene expression biomarkers, we performed causal graph analysis to determine the inter-dependent pathways of these factors on continuous JSN over 24 months (Fig. 2). Baseline PBL COX-2 gene expression and baseline medial BML each independently played a causal role or positively influenced JSN. Moreover, age, sex, and BMI did not show a direct effect on joint space narrowing. However, the open circles adjacent to several predictors, including sex, age, BMI, and osteophytes, indicate that there may be hidden confounders, which may influence their relationships with JSN. These new data demonstrate that the PBL inflammatory COX-2 gene expression and medial $\mathrm{BML}$, independently from other MRI or radiographic features, influence radiographic JSN progression.

\section{Discussion}

In the studies here reported, we analyzed two independent cohorts totaling 447 subjects with symptomatic knee $\mathrm{OA}$ to determine the prognostic value of baseline $\mathrm{PBL}$ 
inflammatory gene expression, semiquantitative osteophyte score, and MRI features, alone and in combination, as biomarkers of radiographic progression in SKOA. Several observations are noteworthy. First, in an expanded NYU cohort and an independent OAI population, we validated our earlier finding that an inflammatory PBL gene expression is a predictive biomarker of OA radiographic progression, consistent with a state of chronic low-grade inflammation in those at risk for progression [15]. Second, consistent with prior literature, medial BML by MRI and osteophyte score by X-ray were associated with radiographic progression at 2 years. Third, and most importantly, we showed that the combinatorial biomarker of the molecular, medial BML, and osteophyte scores markedly increased the predictive value over that of each biomarker alone.

In our analysis of the NYU cohort, we show that baseline medial tibial BMLs were moderately associated with WOMAC and VAS pain, consistent with most prior studies [48, 49]. However, none of the MRI findings were predictive of worsening WOMAC or VAS pain at 2 years. In our study (adjusting for age, sex, and BMI) and in agreement with others [21,50], we observed a strong inverse correlation of baseline medial BMLs with radiographic JSW and a positive correlation with increased JSN over 24 months (Table 3).

Causal analysis of our data indicates that PBL inflammatory gene expression molecular biomarkers and BML are each independently associated with radiographic progression. Therefore, since these features appear to represent discrete pathogenic processes, it is not surprising that the combination of both molecular and MRI biomarkers significantly increases the prediction of radiographic progression. In addition, we show that the addition of osteophyte scores also enhanced the predictive value of the individual PBL and MRI biomarkers. To our knowledge, this is the first study that has shown the improved prognostic capability for knee OA progression based on a combination of MRI imaging (medial BML), molecular, and radiographic findings.

There have been other recent publications that show the predictive value of molecular or imaging biomarkers on progression. A recent FNIH (Foundation of the NIH) study as part of the OA biomarker consortium identified several promising candidates systemic biomarkers such as urine collagen ( $\mathrm{UCTXII}$ and $\mathrm{uCTX1a)}$ ) as predictors of pain and structural worsening of OA [51]. The final baseline model included uCTXII and sNTXI and had an AUC of 0.586. Similar to the unbiased causal analysis of various biomarkers (Fig. 2) in this study, Loeser and associates have also through an unbiased machine learning approach identified BML, osteophytes, and medial meniscal extrusion as potential biomarkers in identifying radiographic $(>0.7 \mathrm{~mm}$ at 48 months $)$ and pain progressors in the OAI cohort [44]. Dunn et al. have studied the peripheral blood methylation status in the radiographic progressors relative to non-progressors in a small cohort of OAI subjects. The epigenetics patterns in peripheral blood mononuclear cells had an AUC of 0.81 in predicting radiographic progressors [52].

What distinguishes our study here is the predictive value of combining molecular biomarkers of inflammation with imaging findings. The combination of biomarkers used together results in predictive values (AUC, odds ratios) for progression that exceed those reported previously for biochemical or imaging alone. In contrast to traditional biochemical markers of cartilage turnover, these data highlight the importance of combining discrete pathogenic events in driving structural progression in OA.

What is the importance of these findings? In addition to shedding light on discrete pathogenic processes, the identification of biomarker predictive of progression is of fundamental importance for the development of new treatment targets through increased efficiencies of trials of disease-modifying agents [51]. There are many obstacles to structure modification studies in OA, including heterogeneity in etiology and the variability in the progression of the disease in clinical trial populations. In ours and prior studies, as many as $25-30 \%$ of knee OA patients will not progress over a 2-year study period, and as few as $25-30 \%$ will progress at a rate that exceeds 0.5 $\mathrm{mm}[11-15,53]$. Therefore, in order to adequately power a clinical trial that demonstrates the efficacy of a structure modifying agent, a need exists to identify biomarkers that can identify a population at risk for disease progression. Therefore, prognostic biomarkers have been sought by industry, frustrated by the challenges of drug development in OA. To date, no single prognostic biomarker has been sufficient, and the predictive value of those described have been modest (e.g., OR of 1.2-1.4) [51]. In our studies, we show that the predictive value of an inflammatory PBL gene expression alone is comparable to that of BML by MRI, with OR in the range of 2-4.

One of the limitations of these findings is that none of the biomarkers studied, alone or in combination, predicted symptomatic worsening at 2 years-a time frame chosen to represent a feasible time period for clinical trials. In part, this may be due to the limited period of observation, as compared, for example, to the 48-month period of follow-up in the FNIH study [44]. We note that BML and cartilage readings are semiquantitative, and it is possible that with more advanced scoring systems, precise evaluation of BML and cartilage volume or size would further improve the progression prediction.

Our studies showed variable effects of BMI on progression, observed in the NYU but not the OAI cohort. 
Demographic differences between the populations included a higher percentage of females in the OAI population, although our sample size were too small to assess the relationship between sex, BMI, and progression. The literature is indeed inconsistent with regard to an independent effect of BMI on radiographic progression of knee OA. While a number publications have shown association of BMI with symptoms and incidence of OA, the literature is mixed regarding a clear association of BMI with OA progression ( [54-57]. An interesting recent article by Wu et al. [58] indicated that the relationship between BMI and progression may also depend on genetics.

. We also note that while the molecular and imaging biomarkers predicted radiographic mISN at 2 years, neither predicted worsening of pain scores over that interval. This is consistent with reports from the OAI that WOMAC scores do not deteriorate significantly among the subjects followed over several years despite radiographic progression for reasons that remain unclear [44]. The presumption remains that slowing structural progression will prove to be a surrogate for improved function and decreased need for joint replacement at future time points. This is a puzzle yet to be demonstrated, most recently in the FGF-18 trial, which resulted in an improvement in total femorotibial joint cartilage thickness after 2 years but no symptomatic relief was reported [59]; a recent study also reported that cartilage thickness loss was associated with small amount of worsening knee pain [60]. Ideally, however, future treatments will provide both symptomatic relief and attenuate disease progression.

\section{Conclusions}

In summary, we demonstrate that the combination of an inflammatory PBL gene expression (molecular), MRI (BML), and radiographic (osteophyte score) biomarkers significantly enhance the predictive value of any individual biomarker in identifying knee OA patients at risk for radiographic progression. The use of predictive biomarkers to identify an OA population at risk for progression is needed for the future design of diseasemodifying $\mathrm{OA}$ drug trials and personalized medicine strategies.

\section{Supplementary information}

Supplementary information accompanies this paper at https://doi.org/10. 1186/s13075-020-02298-6.

Additional file 1.

\section{Abbreviations}

OA: Osteoarthritis; SKOA: Symptomatic knee osteoarthritis; KL grade: Kellgren-Lawrence grade; JSW: Joint space width; JSN: Joint space narrowing; BML: Bone marrow lesion; PBL: Peripheral blood leukocyte cells;
IL-1 $\beta$ : Interleukin 1 beta; TNFa: Tumor necrosis factor alpha; COX2: Cyclooxygenase 2; WOMAC: Western Ontario and McMaster Universities Osteoarthritis index; VAS: Visual analog scale; WORMS: Whole-Organ Magnetic Resonance Imaging Score; BMI: Body mass index; AUC: Area under the receiver operating characteristic curves; OR: Odds ratio

\section{Acknowledgements}

The NYU and OAI cohort biospecimens were collected as part of an $\mathrm{NIH-}$ funded study (National Institute of Arthritis and Musculoskeletal and Skin Diseases grant AR052873 to Dr. Abramson). This work was also supported by the generous contributions of the William and Lynda Steere Foundation. The authors would like to thank the staff and participants of the NYULOH Osteoarthritis Biomarkers Study.

\section{Authors' contributions}

All authors were involved in drafting the article or revising it critically for important intellectual content, and all authors approved the final version to be published. Drs. MA, SK, HZ, and SA had full access to all the data in the study and take responsibility for the integrity of the data and accuracy of the data analysis. MA, SK, JS, PR, and SA contributed to the study conception and design. MA, SK, HZ, JS, GC, JB, PR, LR, JH, and VK contributed to the acquisition of data. MA, SK, HZ, JS, GC, JB, PR, LR, JH, VK, and SA contributed to the analysis and interpretation of data.

\section{Funding}

The NYU and OAl cohort biospecimens were collected as part of an $\mathrm{NIH}$ funded study (National Institute of Arthritis and Musculoskeletal and Skin Diseases grant AR052873 to Dr. Abramson).

This work was also supported by the generous contributions of the William and Lynda Steere Foundation.

This manuscript was prepared using an OAl public use data set and does not necessarily reflect the opinions or views of the OAl investigators, the $\mathrm{NIH}$, or the private funding partners.

\section{Availability of data and materials}

Please contact author for data requests. OAI data are available from OAl.epiuscf.org

Ethics approval and consent to participate

The current study was performed in accordance with the ethical standards of the Helsinki Declaration, and studies (\#i9018 and i12-03682) were approved by the Institutional Review Board (IRB) of NYU School of Medicine. All patients provided written, informed consent to participate in the study.

Consent for publication

Not applicable.

\section{Competing interests}

Drs. Abramson and Attur have patent application (Patent number: 9804175) for the use of inflammatory gene expression biomarkers to identifying patients at risk for knee OA progression.

\section{Author details}

'Department of Medicine, NYU Grossman School of Medicine, New York, NY, USA. ${ }^{2}$ Division of Rheumatology, Rheumatology Research laboratory, NYU Langone Orthopedic Hospital, 301 East 17th Street, Suite 1612, New York, NY 10003, USA. ${ }^{3}$ Applied Bioinformatics Laboratories, NYU Grossman School of Medicine, New York, NY, USA. ${ }^{4}$ Department of Radiology, NYU Grossman School of Medicine, New York, NY, USA. ${ }^{5}$ Division of Musculoskeletal Imaging, Department of Radiology, Hospital of the University of Pennsylvania, Philadelphia, PA 19104, USA. ${ }^{6}$ Duke University School of Medicine, Durham, NC, USA.

Received: 7 February 2020 Accepted: 24 August 2020

Published online: 10 September 2020

References

1. Lawrence RC, Felson DT, Helmick CG, Arnold LM, Choi H, Deyo RA, et al. Estimates of the prevalence of arthritis and other rheumatic conditions in the United States. Part II Arthritis Rheum. 2008;58(1):26-35. 
2. Martel-Pelletier J, Barr AJ, Cicuttini FM, Conaghan PG, Cooper C, Goldring MB, et al. Osteoarthritis. Nat Rev Dis Primers. 2016;2:16072.

3. Deveza LA, Melo L, Yamato TP, Mills K, Ravi V, Hunter DJ. Knee osteoarthritis phenotypes and their relevance for outcomes: a systematic review. Osteoarthr Cartil. 2017;25(12):1926-41.

4. Abramson SB, Attur M. Developments in the scientific understanding of osteoarthritis. Arthritis Res Ther. 2009;11(3):227.

5. Loeser RF, Goldring SR, Scanzello CR, Goldring MB. Osteoarthritis: a disease of the joint as an organ. Arthritis Rheum. 2012;64(6):1697-707.

6. Berenbaum F. Osteoarthritis as an inflammatory disease (osteoarthritis is not osteoarthrosis!). Osteoarthr Cartil. 2013;21(1):16-21.

7. Guermazi A, Hayashi D, Roemer FW, Felson DT. Osteoarthritis: a review of strengths and weaknesses of different imaging options. Rheum Dis Clin N Am. 2013;39(3):567-91.

8. Emrani PS, Katz JN, Kessler CL, Reichmann WM, Wright EA, McAlindon TE, et al. Joint space narrowing and Kellgren-Lawrence progression in knee osteoarthritis: an analytic literature synthesis. Osteoarthr Cartil. 2008;16(8): 873-82

9. Gensburger D, Arlot M, Sornay-Rendu E, Roux JP, Delmas P. Radiologic assessment of age-related knee joint space changes in women: a 4-year longitudinal study. Arthritis Rheum. 2009;61(3):336-43.

10. Driban JB, Stout $A C$, Lo GH, Eaton CB, Price LL, Lu B, et al. Best performing definition of accelerated knee osteoarthritis: data from the Osteoarthritis Initiative. Ther Adv Musculoskelet Dis. 2016;8(5):165-71.

11. Bingham CO 3rd, Buckland-Wright JC, Garnero P, Cohen SB, Dougados M, Adami $\mathrm{S}$, et al. Risedronate decreases biochemical markers of cartilage degradation but does not decrease symptoms or slow radiographic progression in patients with medial compartment osteoarthritis of the knee: results of the two-year multinational knee osteoarthritis structural arthritis study. Arthritis Rheum. 2006;54(11):3494-507.

12. Kumm J, Tamm A, Lintrop M, Tamm A. The prevalence and progression of radiographic knee osteoarthritis over 6 years in a population-based cohort of middle-aged subjects. Rheumatol Int. 2012;32(11):3545-50.

13. Fukui N, Yamane S, Ishida S, Tanaka K, Masuda R, Tanaka N, et al. Relationship between radiographic changes and symptoms or physical examination findings in subjects with symptomatic medial knee osteoarthritis: a three-year prospective study. BMC Musculoskelet Disord. 2010;11:269.

14. Mazzuca SA, Brandt KD, Katz BP, Lane KA, Buckwalter KA. Comparison of quantitative and semiquantitative indicators of joint space narrowing in subjects with knee osteoarthritis. Ann Rheum Dis. 2006;65(1):64-8.

15. Attur M, Krasnokutsky S, Statnikov A, Samuels J, Li Z, Friese O, et al. Lowgrade inflammation in symptomatic knee osteoarthritis: prognostic value of inflammatory plasma lipids and peripheral blood leukocyte biomarkers. Arthritis Rheumatol. 2015;67(11):2905-15.

16. Attur M, Statnikov A, Samuels J, Li Z, Alekseyenko AV, Greenberg JD, et al. Plasma levels of interleukin-1 receptor antagonist (IL1Ra) predict radiographic progression of symptomatic knee osteoarthritis. Osteoarthr Cartil. 2015:23(11):1915-24.

17. Attur M, Belitskaya-Levy I, Oh C, Krasnokutsky S, Greenberg J, Samuels J, et al. Increased interleukin-1beta gene expression in peripheral blood leukocytes is associated with increased pain and predicts risk for progression of symptomatic knee osteoarthritis. Arthritis Rheum. 2011;63(7): 1908-17.

18. Robinson WH, Lepus CM, Wang Q, Raghu H, Mao R, Lindstrom TM, et al. Low-grade inflammation as a key mediator of the pathogenesis of osteoarthritis. Nat Rev Rheumatol. 2016;12(10):580-92.

19. Ramos YF, Bos SD, Lakenberg N, Bohringer S, den Hollander WJ, Kloppenburg M, et al. Genes expressed in blood link osteoarthritis with apoptotic pathways. Ann Rheum Dis. 2014;73(10):1844-53.

20. Hunter DJ, Zhang Y, Niu J, Goggins J, Amin S, LaValley MP, et al. Increase in bone marrow lesions associated with cartilage loss: a longitudinal magnetic resonance imaging study of knee osteoarthritis. Arthritis Rheum. 2006;54(5): 1529-35.

21. Edwards MH, Parsons C, Bruyere O, Petit Dop F, Chapurlat R, Roemer FW, et al. High Kellgren-Lawrence grade and bone marrow lesions predict worsening rates of radiographic joint space narrowing; The SEKOIA Study. J Rheumatol. 2016;43(3):657-65.

22. Crema MD, Nevitt MC, Guermazi A, Felson DT, Wang K, Lynch JA, et al. Progression of cartilage damage and meniscal pathology over 30 months is associated with an increase in radiographic tibiofemoral joint space narrowing in persons with knee OA--the MOST study. Osteoarthr Cartil. 2014;22(10):1743-7.

23. Hunter DJ, Lavalley M, Li J, Bauer DC, Nevitt M, DeGroot J, et al. Biochemical markers of bone turnover and their association with bone marrow lesions. Arthritis Res Ther. 2008;10(4):R102.

24. Garnero P, Peterfy C, Zaim S, Schoenharting M. Bone marrow abnormalities on magnetic resonance imaging are associated with type II collagen degradation in knee osteoarthritis: a three-month longitudinal study. Arthritis Rheum. 2005;52(9):2822-9.

25. Deveza LA, Kraus VB, Collins JE, Guermazi A, Roemer FW, Bowes M, et al. Association between biochemical markers of bone turnover and bone changes on imaging: data from the osteoarthritis initiative. Arthritis Care Res (Hoboken). 2017:69(8):1179-91.

26. Deveza LA, Kraus VB, Collins JE, Guermazi A, Roemer FW, Nevitt MC, et al. Is synovitis detected on non-contrast-enhanced magnetic resonance imaging associated with serum biomarkers and clinical signs of effusion? Data from the Osteoarthritis Initiative. Scand J Rheumatol. Scan J Rheumatol. 2018; 47(3):235-42.

27. Dam EB, Loog M, Christiansen C, Byrjalsen I, Folkesson J, Nielsen M, et al. Identification of progressors in osteoarthritis by combining biochemical and MRI-based markers. Arthritis Res Ther. 2009;11(4):R115.

28. Arden N, Richette P, Cooper C, Bruyere O, Abadie E, Branco J, et al. Can we identify patients with high risk of osteoarthritis progression who will respond to treatment? A focus on biomarkers and frailty. Drugs Aging 2015;32(7):525-35.

29. Krasnokutsky S, Oshinsky C, Attur M, Ma S, Zhou H, Zheng F, et al. Serum urate levels predict joint space narrowing in non-gout patients with medial knee osteoarthritis. Arthritis Rheumatol. 2017;69(6):1213-20.

30. Kellgren JH, Lawrence JS. Radiological assessment of osteo-arthrosis. Ann Rheum Dis. 1957;16(4):494-502

31. Peterfy CG, Guermazi A, Zaim S, Tirman PF, Miaux Y, White D, et al. WholeOrgan Magnetic Resonance Imaging Score (WORMS) of the knee in osteoarthritis. Osteoarthr Cartil. 2004;12(3):177-90.

32. Vignon E, Brandt KD, Mercier C, Hochberg M, Hunter D, Mazzuca S, et al. Alignment of the medial tibial plateau affects the rate of joint space narrowing in the osteoarthritic knee. Osteoarthr Cartil. 2010;18(11):1436-40.

33. Attur M, Zhou H, Samuels J, Krasnokutsky S, Yau M, Scher JU, et al. Interleukin 1 receptor antagonist (IL1RN) gene variants predict radiographic severity of knee osteoarthritis and risk of incident disease. Ann Rheum Dis. 2019;79:400-7.

34. Peterfy CG, Schneider E, Nevitt M. The osteoarthritis initiative: report on the design rationale for the magnetic resonance imaging protocol for the knee. Osteoarthr Cartil. 2008;16(12):1433-41.

35. Hunter DJ, Guermazi A, Lo GH, Grainger AJ, Conaghan PG, Boudreau RM, et al. Evolution of semi-quantitative whole joint assessment of knee OA: MOAKS (MRI osteoarthritis knee score). Osteoarthr Cartil. 2011;19(8):990-1002.

36. Livak KJ, Schmittgen TD. Analysis of relative gene expression data using real-time quantitative PCR and the 2(-Delta Delta C(T)) method. Methods. 2001;25(4):402-8.

37. Vapnik VN. An overview of statistical learning theory. IEEE Trans Neural Netw. 1999:10(5):988-99.

38. Braga-Neto UM, Dougherty ER. Is cross-validation valid for small-sample microarray classification? Bioinformatics. 2004;20(3):374-80.

39. Fawcett T. An introduction to ROC analysis. Pattern Recogn Lett. 2006;27(8): $861-74$

40. DeLong ER, DeLong DM, Clarke-Pearson DL. Comparing the areas under two or more correlated receiver operating characteristic curves: a nonparametric approach. Biometrics. 1988;44(3):837-45.

41. Benjamini $Y$, Hochberg Y. Controlling the false discovery rate: a practical and powerful approach to multiple testing. J R Stat Soc Ser B. 1995;57(1): 289-300.

42. Cooper C, Adachi JD, Bardin T, Berenbaum F, Flamion B, Jonsson H, et al. How to define responders in osteoarthritis. Curr Med Res Opin. 2013;29(6): 719-29.

43. Nevitt MC, Peterfy C, Guermazi A, Felson DT, Duryea J, Woodworth T, et al Longitudinal performance evaluation and validation of fixed-flexion radiography of the knee for detection of joint space loss. Arthritis Rheum. 2007:56(5):1512-20.

44. Nelson AE, Fang F, Arbeeva L, Cleveland RJ, Schwartz TA, Callahan LF, et al. A machine learning approach to knee osteoarthritis phenotyping: data from the FNIH Biomarkers Consortium. Osteoarthr Cartil. 2019;27(7):994-1001. 
45. Felson DT, Gale DR, Elon Gale M, Niu J, Hunter DJ, Goggins J, et al. Osteophytes and progression of knee osteoarthritis. Rheumatology (Oxford). 2005;44(1):100-4.

46. Nagaosa Y, Lanyon P, Doherty M. Characterisation of size and direction of osteophyte in knee osteoarthritis: a radiographic study. Ann Rheum Dis. 2002;61(4):319-24

47. de Lange-Brokaar BJ, Bijsterbosch J, Kornaat PR, Yusuf E, loan-Facsinay A, Zuurmond AM, et al. Radiographic progression of knee osteoarthritis is associated with MRI abnormalities in both the patellofemoral and tibiofemoral joint. Osteoarthr Cartil. 2016;24(3):473-9.

48. de Lange-Brokaar BJ, loan-Facsinay A, Yusuf E, Kroon HM, Zuurmond AM, Stojanovic-Susulic V, et al. Evolution of synovitis in osteoarthritic knees and its association with clinical features. Osteoarthr Cartil. 2016;24(11):1867-74.

49. Alliston T, Hernandez CJ, Findlay DM, Felson DT, Kennedy OD. Bone marrow lesions in osteoarthritis: what lies beneath. J Orthop Res. 2018:36(7):1818-25.

50. Driban JB, Lo GH, Lee JY, Ward RJ, Miller E, Pang J, et al. Quantitative bone marrow lesion size in osteoarthritic knees correlates with cartilage damage and predicts longitudinal cartilage loss. BMC Musculoskelet Disord. 2011;12: 217.

51. Kraus VB, Collins JE, Hargrove D, Losina E, Nevitt M, Katz JN, et al. Predictive validity of biochemical biomarkers in knee osteoarthritis: data from the FNIH OA biomarkers consortium. Ann Rheum Dis. 2017:76(1):186-95.

52. Dunn MC, Nevitt MC, Lynch JA, Jeffries MA. A pilot study of peripheral blood DNA methylation models as predictors of knee osteoarthritis radiographic progression: data from the Osteoarthritis Initiative (OAI). Sci Rep. 2019;9(1):16880

53. Parsons CM, Judge A, Leyland K, Bruyere O, Petit Dop F, Chapurlat R, et al. Novel approach to estimate osteoarthritis progression: use of the reliable change index in the evaluation of joint space loss. Arthritis Care Res (Hoboken). 2019;71(2):300-7.

54. Le Graverand MP, Brandt K, Mazzuca SA, Raunig D, Vignon E. Progressive increase in body mass index is not associated with a progressive increase in joint space narrowing in obese women with osteoarthritis of the knee. Ann Rheum Dis. 2009;68(11):1734-8

55. Niu J, Zhang YQ, Torner J, Nevitt M, Lewis CE, Aliabadi P, et al. Is obesity a risk factor for progressive radiographic knee osteoarthritis? Arthritis Rheum. 2009:61(3):329-35.

56. Yusuf E, Bijsterbosch J, Slagboom PE, Rosendaal FR, Huizinga TW, Kloppenburg M. Body mass index and alignment and their interaction as risk factors for progression of knees with radiographic signs of osteoarthritis. Osteoarthr Cartil. 2011;19(9):1117-22.

57. Zhou ZY, Liu YK, Chen HL, Liu F. Body mass index and knee osteoarthritis risk: a dose-response meta-analysis. Obesity (Silver Spring). 2014;22(10): $2180-5$.

58. Wu X, Kondragunta V, Kornman KS, Wang HY, Duff GW, Renner JB, et al. IL-1 receptor antagonist gene as a predictive biomarker of progression of knee osteoarthritis in a population cohort. Osteoarthr Cartil. 2013;21(7):930-8.

59. Hochberg MC, Guermazi A, Guehring H, Aydemir A, Wax S, FleuranceauMorel P, et al. Effect of intra-articular sprifermin vs placebo on femorotibial joint cartilage thickness in patients with osteoarthritis: the FORWARD randomized clinical trial. JAMA. 2019;322(14):1360-70.

60. Bacon K, LaValley MP, Jafarzadeh SR, Felson D. Does cartilage loss cause pain in osteoarthritis and if so, how much? Ann Rheum Dis. 2020;79(8): 1105-10.

\section{Publisher's Note}

Springer Nature remains neutral with regard to jurisdictional claims in published maps and institutional affiliations.

Ready to submit your research? Choose BMC and benefit from:

- fast, convenient online submission

- thorough peer review by experienced researchers in your field

- rapid publication on acceptance

- support for research data, including large and complex data types

- gold Open Access which fosters wider collaboration and increased citations

- maximum visibility for your research: over $100 \mathrm{M}$ website views per year

At BMC, research is always in progress.

Learn more biomedcentral.com/submissions 